\title{
ON BAZILEVIČ FUNCTIONS
}

\author{
BY \\ D. K. THOMAS( $\left.{ }^{1}\right)$
}

1. In 1955 E. Bazilevič [1] introduced a class of functions $f(z)$ in $|z|<1$ and defined by the following relation:

$$
f(z)=\left\{\frac{\beta}{1+\alpha^{2}} \int_{0}^{z}(h(\zeta)-\alpha i) \zeta^{\left[-\alpha \beta i /\left(1+\alpha^{2}\right)\right]-1} g(\zeta)^{\beta /\left(1+\alpha^{2}\right)} d \zeta\right\}^{(1+\alpha i) / \beta},
$$

where $h(z)=1+\sum_{n=1}^{\infty} c_{n} z^{n}$ satisfies $\operatorname{Re} h(z)>0$ in $|z|<1, g(z)$ is starlike (w.r.t. origin) in $|z|<1, \alpha$ is any real number and $\beta>0\left({ }^{2}\right)$. He was able to show that each such function is univalent in $|z|<1$. Pommerenke [10] has also given a proof of this.

Little is known about the class of functions defined by (1) and indeed little is known about the subclass of those functions for which $\alpha=0$. If we put $\alpha=0$ in (1) then we have

$$
f(z)=\left\{\beta \int_{0}^{z} h(\zeta) \zeta^{-1} g(\zeta)^{\beta} d \zeta\right\}^{1 / \beta},
$$

and on differentiating this expression we obtain

$$
z f^{\prime}(z)=f(z)^{1-\beta} g(z)^{\beta} h(z)
$$

where

$$
\operatorname{Re} \frac{z f^{\prime}(z)}{f(z)^{1-\beta} g(z)^{\beta}}>0 \quad(|z|<1) .
$$

We shall call a function satisfying (3) a Bazilevič function of type $\beta$. We notice that if $\beta=1$ we have the class of close-to-convex functions, and in this case (3) has a geometrical interpretation [6]. It is not clear whether any geometrical meaning can be deduced from (3) for any other value of $\beta$.

The Bieberbach conjecture is still unsettled for Bazilevič functions, the only contribution so far being made by J. Zamorski [12] who showed that the conjecture is valid when $\beta=1 / N, N$ being a positive integer.

In this paper we shall be concerned mainly with problems of bounded Bazilevič functions, to which we now turn.

2. Let $f(z)=z+a_{2} z^{2}+\cdots$ be univalent and bounded in $|z|<1$. If further $f(z)$ is close-to-convex in $|z|<1$ then, as was shown by Clunie and Pommerenke [3], the

Received by the editors March 28, 1967.

(1) This work was carried out under the supervision of Professor J. G. Clunie at Imperial College, London while the author was working for a Ph.D. degree in the University of London.

(2) All powers are meant as principal values. 
coefficients of $f(z)$ satisfy $a_{n}=O(1 / n)$ and this is best possible. In this section we shall extend the method of Clunie and Pommerenke and prove $a_{n}=O(1 / n)$ remains valid for the class of Bazilevič functions of type $\beta$.

THEOREM 1. Let $f(z)=z+\sum_{n=2}^{\infty} a_{n} z^{n}$ be a Bazilevic function of type $\beta$ and let $|f(z)|<1$ in $|z|<1$. Then $\left|a_{n}\right| \leqq K(\beta) / n,(n=2,3, \ldots)$, where $K(\beta)$ is a constant depending only upon $\beta$.

Proof. In (2) we write, with $z=r e^{i \theta}$

$$
z f^{\prime}(z)=2 f(z)^{1-\beta} g(z)^{\beta} \operatorname{Re} h(z)-f(z)^{1-\beta} g(z)^{\beta} \overline{h(z)} .
$$

Then since

(4) gives

$$
n a_{n}=\frac{1}{2 \pi r^{n}} \int_{0}^{2 \pi} z f^{\prime}(z) e^{-i n \theta} d \theta
$$

$$
\begin{aligned}
n a_{n}= & \frac{1}{\pi r^{n}} \int_{0}^{2 \pi} f(z)^{1-\beta} g(z)^{\beta} \operatorname{Re} h(z) e^{-i n \theta} d \theta \\
& -\frac{1}{2 \pi r^{n}} \int_{0}^{2 \pi} f(z)^{1-\beta} g(z)^{\beta} \overline{h(z)} e^{-i n \theta} d \theta
\end{aligned}
$$

Therefore we write

$$
\begin{aligned}
n\left|a_{n}\right| & \leqq \frac{1}{\pi r^{n}} \int_{0}^{2 \pi}\left|f(z)^{1-\beta} g(z)^{\beta}\right| \operatorname{Re} h(z) d \theta+\frac{1}{2 \pi r^{n}}\left|\int_{0}^{2 \pi} f(z)^{1-\beta} g(z)^{\beta} \overline{h(z)} e^{-i n \theta} d \theta\right| \\
& =I_{1}(r)+I_{2}(r) .
\end{aligned}
$$

By hypothesis $|f(z)|^{1-\beta}<1$ if $0<\beta \leqq 1$. Also it follows from a 'distortion theorem' for univalent functions $\left[5, \mathrm{p}\right.$. 3] that $|f(z)|^{1-\beta} \leqq(4 / r)^{\beta-1}$ if $\beta \geqq 1$. Thus

$$
I_{1}(r) \leqq \frac{K_{1}(\beta)}{\pi r^{n}} \int_{0}^{2 \pi}|g(z)|^{\beta} \operatorname{Re} h(z) d \theta
$$

where $K_{1}(\beta)=\max \left\{1,(4 / r)^{\beta-1}\right\}, r$ fixed, $0<r<1$.

Since $\operatorname{Re} h(z)>0$ in $|z|<1$ we obtain

$$
\begin{aligned}
I_{1}(r) & \leqq \frac{K_{1}(\beta)}{\pi r^{n}} \operatorname{Re} \int_{0}^{2 \pi}|g(z)|^{\beta} h(z) d \theta \\
& =\frac{K_{1}(\beta)}{\pi r^{n}} \operatorname{Re} \int_{0}^{2 \pi} z f^{\prime}(z) f(z)^{\beta-1} \exp (-i \beta \arg g(z)) d \theta
\end{aligned}
$$

on using (2). On integrating this expression by parts we deduce that

$$
I_{1}(r) \leqq \frac{K_{1}(\beta)}{\pi r^{n}} \operatorname{Re} \int_{0}^{2 \pi} f(z) \exp (-i \beta \arg g(z)) d_{\theta}\{\arg g(z)\} \leqq \frac{2 K_{1}(\beta)}{r^{n}},
$$

since $\int_{0}^{2 \pi} d_{\theta}\{\arg g(z)\}=2 \pi$ and $|f(z)|<1$. 
On taking complex conjugates we obtain

$$
\begin{aligned}
I_{2}(r) & =\frac{1}{2 \pi r^{n}}\left|\int_{0}^{2 \pi} \overline{f(z)^{1}-\beta} \overline{g(z)^{\beta}} h(z) e^{i n \theta} d \theta\right| \\
& =\frac{1}{2 \pi r^{n}}\left|\int_{0}^{2 \pi} \overline{f(z)^{1}-\beta} z f^{\prime}(z) f(z)^{\beta-1} \exp (-2 i \beta \arg g(z)) e^{i n \theta} d \theta\right|
\end{aligned}
$$

from (2). Hence

$$
\begin{aligned}
I_{2}(r) & =\frac{1}{2 \pi r^{n}}\left|\int_{0}^{2 \pi} z f^{\prime}(z) \exp (2 i(\beta-1) \arg f(z)) \exp (-2 i \beta \arg g(z)) e^{i n \theta} d \theta\right| \\
& =\frac{1}{2 \pi r^{2 n}}\left|\int_{0}^{2 \pi} z^{n+1} f^{\prime}(z) \exp (2 i(\beta-1) \arg f(z)) \exp (-2 i \beta \arg g(z)) d \theta\right|
\end{aligned}
$$

Define

$$
f_{n}(z)=\int_{0}^{z} \zeta^{n} f^{\prime}(\zeta) d \zeta
$$

Then integrating (6) by parts we have

$$
\begin{aligned}
I_{2}(r)= & \frac{1}{2 \pi r^{2 n}}\left|\int_{0}^{2 \pi} f_{n}(z) d_{\theta}\{\exp (2 i(\beta-1) \arg f(z)) \exp (-2 i \beta \arg g(z))\}\right| \\
\leqq & \frac{|\beta-1|}{\pi r^{2 n}}\left|\int_{0}^{2 \pi} f_{n}(z) \exp (2 i(\beta-1) \arg f(z)) \exp (-2 i \beta \arg g(z)) \operatorname{Re} z f^{\prime}(z)\right| f(z) d \theta \mid \\
& +\frac{\beta}{\pi r^{2 n}}\left|\int_{0}^{2 \pi} f_{n}(z) \exp (2 i(\beta-1) \arg f(z)) \exp (-2 i \beta \arg g(z)) d_{\theta}\{\arg g(z)\}\right| \\
= & J_{1}(r)+J_{2}(r), \text { say. }
\end{aligned}
$$

From (7) we have, on integrating by parts,

$$
\left|f_{n}(z)\right| \leqq 2 r^{n},
$$

and since $g(z)$ is starlike we deduce that

$$
J_{2}(r) \leqq 4 \beta / r^{n} .
$$

Now

$$
J_{1}(r) \leqq \frac{|\beta-1|}{\pi r^{2 n}} \int_{0}^{2 \pi}\left|f_{n}(z)\right|\left|\frac{z f^{\prime}(z)}{f(z)}\right| d \theta .
$$

Hence by the Schwarz inequality

$$
\begin{aligned}
J_{1}(r)^{2} & \leqq \frac{(\beta-1)^{2}}{\pi^{2} r^{4 n}} \int_{0}^{2 \pi}\left|f_{n}(z)\right|^{2} d \theta \int_{0}^{2 \pi}\left|\frac{z f^{\prime}(z)}{f(z)}\right|^{2} d \theta \\
& \leqq \frac{16(\beta-1)^{2}}{\pi^{2} r^{4 n}} \int_{0}^{2 \pi}\left|f_{n}(z)\right|^{2} d \theta \int_{0}^{2 \pi}\left|f^{\prime}(z)\right|^{2} d \theta
\end{aligned}
$$


on using a 'distortion theorem'. In order to estimate these last two integrals we require the following two lemmas:

LEMMA 1. With the above notation and with $|f(z)|<1$,

$$
\int_{0}^{2 \pi}\left|f_{n}(z)\right|^{2} d \theta \leqq \frac{\pi r^{2 n}}{2 n} \quad\left(z=r e^{i \theta}\right) .
$$

LEMMA 2. For any normalized univalent function satisfying $|f(z)|<1$,

$$
\int_{0}^{2 \pi}\left|f^{\prime}(z)\right|^{2} d \theta \leqq \frac{32 \pi}{r^{3} e(1-r)} \quad\left(z=r e^{i \theta}\right)
$$

For a proof of Lemma 1 see Clunie and Pommerenke [3]. Lemma 2 is well known.

We now apply Lemmas 1 and 2 in (10) and obtain

$$
J_{1}(r) \leqq \frac{16|\beta-1|}{\sqrt{e} r^{n+3 / 2} \sqrt{ }(n(1-r))} .
$$

Choosing $r=1-1 / n+1$ we have from (5), (9), and (11) $n\left|a_{n}\right| \leqq K(\beta)$, where $K(\beta)=$ $2 e K_{1}(\beta)+64 \sqrt{ } e|\beta-1|+4 \beta e$. This completes the proof of Theorem 1 .

Note that when $\beta=1$, that is for close-to-convex functions, $K(\beta)=6 e$. However an examination of the proof of Theorem 1 shows that $J_{1}(r)$ does not arise in this case. This enables us to let $r$ tend to 1 in (5) and (9) giving $K(1)=6$ which is a slight improvement of the constant $K(1)=9.3$ given in [3].

3. Let $C(r)$ denote the closed curve which is the image of the circle $|z|=r<1$ under the mapping $w=f(z)$, and let $L(r)$ denote the length of $C(r)$. In [11] it was shown that if $f(z)$ is a bounded close-to-convex function in $|z|<1$, then

$$
L(r)=O\{\log [1 /(1-r)]\} \quad \text { as } r \rightarrow 1,
$$

and $O$ cannot be replaced by $o$ in general. In this section we shall extend this result and prove the following:

TheOREM 2. Let $f(z)=z+\sum_{n=2}^{\infty} a_{n} z^{n}$ be a Bazilevič function of type $\beta$ and let $|f(z)|<1$ in $|z|<1$. Then

$$
L(r)=O\{\log [1 /(1-r)]\} \quad \text { as } r \rightarrow 1,
$$

the $O$ indicating a constant depending only upon $\beta$.

Proof. We have, using (2),

$$
\begin{aligned}
L(r) & =\int_{0}^{2 \pi}\left|z f^{\prime}(z)\right| d \theta=\int_{0}^{2 \pi}\left|f(z)^{1-\beta} g(z)^{\beta} h(z)\right| d \theta \\
& \leqq K_{1}(\beta) \int_{0}^{2 \pi}\left|g(z)^{\beta} h(z)\right| d \theta \quad\left(z=r e^{i \theta}\right)
\end{aligned}
$$


as in the proof of Theorem 1. Thus

$$
\begin{aligned}
L(r) \leqq & K_{1}(\beta)\left\{\beta \int_{0}^{r} \int_{0}^{2 \pi}\left|g(z)^{\beta-1} g^{\prime}(z) h(z)\right| d \theta d \rho\right. \\
& \left.\quad+\int_{0}^{r} \int_{0}^{2 \pi}\left|g(z)^{\beta} h^{\prime}(z)\right| d \theta d \rho\right\} \quad\left(z=\rho e^{i \theta}\right) \\
= & K_{1}(\beta)\left[I_{1}(r)+I_{2}(r)\right] .
\end{aligned}
$$

Since $g(z)$ is starlike we may write

$$
z g^{\prime}(z)=g(z) \phi(z),
$$

where $\phi(z)=1+\sum_{n=1}^{\infty} d_{n} z^{n}$ and $\operatorname{Re} \phi(z)>0$ in $|z|<1$. Then

$$
I_{1}(r) \leqq \beta K_{2}(\beta) \int_{0}^{r} \int_{0}^{2 \pi}\left|f^{\prime}(z) \phi(z)\right| d \theta d \rho,
$$

where we have used (2), (13) and a 'distortion theorem', and where $K_{2}(\beta)=$ $\max \left\{1,(4 / r)^{1-\beta}\right\}, r$ fixed; $0<r<1$. But the expression $\int_{0}^{r} \int_{0}^{2 \pi}\left|f^{\prime}(z) \phi(z)\right| d \theta d \rho$ is just $J_{1}$ in [11, Theorem 2], and so using this result

$$
I_{1}(r) \leqq 2(2 \pi)^{1 / 2} \beta K_{2}(\beta)\left(\frac{1}{r} \log \frac{1+r}{1-r}\right)^{1 / 2}
$$

Using a well-known result for functions of positive real part (see e.g. [4, p. 337]) we have

$$
\begin{aligned}
I_{2}(r) & =\int_{0}^{r} \int_{0}^{2 \pi}\left|g(z)^{\beta} h^{\prime}(z)\right| d \theta d \rho \\
& =2 \operatorname{Re} \int_{0}^{r} \int_{0}^{2 \pi}|g(z)|^{\beta} h(z) d \theta d \rho /\left(1-\rho^{2}\right) \\
& =2 \beta \operatorname{Re} \int_{0}^{r} \int_{0}^{2 \pi} f(z)^{\beta} \exp (-i \beta \arg g(z)) d_{\theta}\{\arg g(z)\} d \rho /\left(1-\rho^{2}\right) \quad\left(z=\rho e^{i \theta}\right)
\end{aligned}
$$

where we have used (2) and integrated by parts. Thus, since $g(z)$ is starlike, we deduce $I_{2}(r) \leqq 2 \pi \beta \log [(1+r) /(1-r)]$. Hence from the estimates for $I_{1}(r)$ and $I_{2}(r)$ we obtain from $(12) L(r) \leqq K(\beta) \log [1 /(1-r)], 0<r<1$. This completes the proof of Theorem 2.

In a similar manner we can prove the following results for Bazilevič functions of type $\beta, 0<\beta \leqq 1$.

THEOREM 3. Let $f(z)$ be a Bazilevič function of type $\beta, 0<\beta \leqq 1$, and let

Then

$$
M(r)=\max _{|z|=r}\left|f\left(r e^{i \theta}\right)\right|, \quad 0<r<1 .
$$

$$
L(r) \leqq K(\beta) M(r) \log [1 /(1-r)] .
$$

Also if $M(r) \leqq(1-r)^{-\alpha}$ we have

(a) $L(r) \leqq K(\alpha, \beta)(1-r)^{-\alpha}$ for $0<\alpha \leqq 2$,

(b) $n\left|a_{n}\right| \leqq K(\alpha, \beta) n^{\alpha}$ for $0 \leqq \alpha \leqq 2$. 
Note. Since $n\left|a_{n}\right| \leqq\left(1 / 2 \pi r^{n}\right) L(r)$, (b) follows at once from (a) for $0<\alpha \leqq 2$. The case $\alpha=0$ is covered in Theorem 1 .

4. In this section we shall introduce the classes of mappings of $|z|>1$ onto domains containing $\infty$ that are analogous to the classes of regular Bazilevič functions of type $\beta$.

Let $f(z)=z+\sum_{n=0}^{\infty} a_{n} z^{-n}$ be regular in $1<|z|<\infty$ and $g(z)=z+\sum_{n=0}^{\infty} b_{n} z^{-n}$ be regular and starlike in $1<|z|<\infty$. Then $f(z)$ is called a meromorphic Bazilevič function of type $\beta$ if there exists a function $g(z)$ satisfying

$$
\operatorname{Re} \frac{z f^{\prime}(z)}{f(z)^{1-\beta} g(z)^{\beta}}>0 \quad \text { for }|z|>1
$$

where $\beta$ is any real number $\left({ }^{3}\right)$.

We define this class for all real $\beta$ as opposed to $\beta>0$, since the latter restriction was imposed in the regular case to ensure that the function was univalent [1]. In the exterior case a function $f(z)$ defined by (14) need not be univalent, for when $\beta=1$ it is easy to construct such a function (see e.g. [8]).

Although our definition is for all real $\beta$, the results we obtain are valid only for $0 \leqq \beta \leqq 1$. We begin by proving:

THEOREM 4. Let both $f(z)=z+\sum_{n=0}^{\infty} a_{n} z^{-n}$ and $g(z)=z+\sum_{n=0}^{\infty} b_{n} z^{-n}$ be regular, not equal to zero and univalent in $1<|z|<\infty$, and let (14) be satisfied for $0 \leqq \beta \leqq 1$. Then with $z=r e^{i \theta}$, and $r>1$

$$
L(r)=r \int_{0}^{2 \pi}\left|f^{\prime}\left(r e^{i \theta}\right)\right| d \theta=O\left(\log \frac{1}{1-r^{-1}}\right) \quad \text { as } r \rightarrow 1 .
$$

REMARKS. 1. Although we have the stronger hypothesis that $f(z)$ is univalent for $1<|z|<\infty$, we have weakened the hypothesis that $g(z)$ is starlike.

2. When $\beta=1$, this theorem is identical to [8, Satz 8].

Proof. Writing (14) in terms of a function $\chi(z)=1+\sum_{n=1}^{\infty} c_{n} z^{-n}$ of positive real part,

$$
L(r)=\int_{0}^{2 \pi}\left|f(z)^{1-\beta} g(z)^{\beta} \chi(z)\right| d \theta \quad\left(z=r e^{i \theta}\right) .
$$

Since $f(z)$ and $g(z)$ are univalent and different from zero, it follows that $\left|f(z)^{1-\beta} g(z)^{\beta}\right| \leqq(r+3)[7$, Vol II, p. 25] whenever $0 \leqq \beta \leqq 1$. Thus

$$
L(r) \leqq(r+3) \int_{0}^{2 \pi}|\chi(z)| d \theta
$$

and this last integral is easily estimated to give the required result.

$\left({ }^{3}\right)$ Again all powers are meant as principal values. 
5. In [8] Pommerenke showed that for meromorphic close-to-convex functions $f(z)=z+\sum_{n=0}^{\infty} a_{n} z^{-n}$ i.e. when $\beta=1$ in (14), the coefficients $a_{n}$ satisfy $n a_{n}=O(1)$. In [9] he produced an example to show that in general $O$ cannot be replaced by $o$. In view of Theorem 2 and the fact that the coefficient property just mentioned holds for both meromorphic and regular bounded close-to-convex functions, it might be thought that this would be valid for functions defined by (14). It is an immediate consequence of Theorem 4 that $n a_{n}=O(\log n)$. Although we are not able to remove the logarithmic factor completely, we improve this result in the following theorem.

THEOREM 5. Let $f(z)=z+\sum_{n=0}^{\infty} a_{n} z^{-n}$ be regular, univalent and not equal to zero in $1<|z|<\infty$. Let $g(z)=z+\sum_{n=0}^{\infty} b_{n} z^{-n}$ be regular, not equal to zero and starlike in $1<|z|<\infty$, and let (14) be satisfied for $0 \leqq \beta \leqq 1$. Then

$$
n a_{n}=O\left\{(\log n)^{1 / 2}\right\}, \quad n \geqq 2,
$$

where the $O$ indicates a constant depending only upon $\beta$.

Proof. We employ the method of Theorem 1. Cauchy's integral formula gives for $n \geqq 1$

$$
n a_{n}=\frac{-r^{n+1}}{2 \pi} \int_{0}^{2 \pi} z f^{\prime}(z) e^{i(n+1) \theta} d \theta \quad\left(z=r e^{i \theta}\right)
$$

Using the techniques of Theorem 1, it follows that

$$
\begin{aligned}
n\left|a_{n}\right| \leqq & \frac{r^{n+1}}{\pi} \int_{0}^{2 \pi}\left|f(z)^{1-\beta} g(z)^{\beta}\right| \operatorname{Re} \chi(z) d \theta \\
& +\frac{r^{n+1}}{2 \pi}\left|\int_{0}^{2 \pi} f(z)^{1-\beta} g(z)^{\beta} \overline{h(z)} e^{i(n+1) \theta} d \theta\right|,
\end{aligned}
$$

where $\chi(z)=1+\sum_{n=1}^{\infty} c_{n} z^{-n}$ and $\operatorname{Re} \chi(z)>0$ in $1<|z|<\infty$. Write this last expression as $I_{1}(r)+I_{2}(r)$.

Since $\left|f(z)^{1-\beta} \mathrm{g}(\mathrm{z})^{\beta}\right| \leqq r+3$ for $0 \leqq \beta \leqq 1$ we have

$$
I_{1}(r) \leqq(r+3) \frac{r^{n+1}}{\pi} \int_{0}^{2 \pi} \operatorname{Re} h(z) d \theta \leqq 2(r+3) r^{n+1} \quad\left(z=r e^{i \theta}\right)
$$

by the mean value theorem for harmonic functions.

Taking the complex conjugate of the integrand of $I_{2}(r)$ we have

$$
\begin{aligned}
& I_{2}(r)=\frac{r^{n+1}}{2 \pi}\left|\int_{0}^{2 \pi} \overline{f(z)^{1}-\beta} \overline{g(z)^{\beta}} h(z) e^{-i(n+1) \theta} d \theta\right| \\
& =\frac{r^{n+1}}{2 \pi}\left|\int_{0}^{2 \pi} z f^{\prime}(z) \exp (-2 i(1-\beta) \arg f(z)) \exp (-2 i \beta \arg g(z)) e^{-i(n+1) \theta} d \theta\right|
\end{aligned}
$$


on using (14)

$$
=\frac{r^{2(n+1)}}{2 \pi}\left|\int_{0}^{2 \pi} \mathscr{F}_{n}(z) d_{\theta}\{\exp (-2 i(1-\beta) \arg f(z)) \exp (-2 i \beta \arg g(z))\}\right|
$$

where

$$
\mathscr{F}_{n}(z)=\int_{\infty}^{z} \zeta^{-n} f^{\prime}(\zeta) d \zeta, \quad n \geqq 2 .
$$

Expanding (16) we have

$$
\begin{aligned}
I_{2}(r) \leqq & \frac{\beta r^{2(n+1)}}{\pi}\left|\int_{0}^{2 \pi} \mathscr{F}_{n}(z) \exp (-2 i(1-\beta) \arg f(z)) \exp (-2 i \arg g(z)) \operatorname{Re} \frac{z g^{\prime}(z)}{g(z)} d \theta\right| \\
& +\frac{r^{2(n+1)}}{\pi} \mid(1-\beta) \int_{0}^{2 \pi} \mathscr{F}_{n}(z) \exp (-2 i(1-\beta) \arg f(z)) \\
= & \quad \times \exp (-2 i \arg g(z)) \operatorname{Re} \frac{z f^{\prime}(z)}{f(z)} d \theta \mid
\end{aligned}
$$

Since $g(z)$ is starlike in $1<|z|<\infty, \operatorname{Re}\left[z g^{\prime}(z) / g(z)\right]>0$ in $1<|z|<\infty$, and so from the mean value theorem for harmonic functions it follows that

$$
J_{1}(r) \leqq 2 \beta r^{2(n+1)} \max _{|z|=r}\left|\mathscr{F}_{n}(z)\right| .
$$

But on integrating (17) by parts we find that $\left|\mathscr{F}_{n}(z)\right| \leqq 2(r+3) r^{-n}$, and hence

$$
J_{1}(r) \leqq 4 \beta(r+3) r^{n+2} .
$$

To estimate $J_{2}(r)$ we apply the Schwarz inequality as follows:

$$
J_{2}(r) \leqq \frac{(1-\beta)}{\pi} r^{2(n+1)}\left(\int_{0}^{2 \pi}\left|\mathscr{F}_{n}(z)\right|^{2} d \theta\right)^{1 / 2}\left(\int_{0}^{2 \pi}\left|\frac{z f^{\prime}(z)}{f(z)}\right|^{2} d \theta\right)^{1 / 2} .
$$

The first integral in this expression can be estimated by establishing a result similar to Lemma 1. The proof, which we omit, is similar to that employed in [3]. We state this result as

Lemma 3. If $\mathscr{F}_{n}(z)$ is defined by (17) then

$$
\frac{1}{2 \pi} \int_{0}^{2 \pi}\left|\mathscr{F}_{n}(z)\right|^{2} d \theta \leqq\left(\frac{n+1}{n-1}\right)^{2} \frac{1}{4 n} \quad \text { for } n \geqq 2 .
$$

It only remains now to estimate the second integral in (19). Write $f(z)=1 / F\left(z^{-1}\right)$. Then $F(\zeta)$ is regular and univalent in $|\zeta|<1$ and

$$
\frac{z f^{\prime}(z)}{f(z)}=\frac{\zeta F^{\prime}(\zeta)}{F(\zeta)}
$$

where $z=r e^{i \theta}, \zeta=1 / z=\operatorname{Re}^{i \phi}$. Thus it is enough to estimate the integral

$$
\int_{0}^{2 \pi}\left|\frac{\zeta F^{\prime}(\zeta)}{F(\zeta)}\right|^{2} d \phi
$$


for regular univalent functions $F(\zeta)$ in $|\zeta|<1$. This integral is of great interest in the theory of univalent functions, the correct rate of growth being unknown at present. However an estimate for (20) which seems to be the best to date is the following:

$$
\int_{0}^{2 \pi}\left|\frac{\zeta F^{\prime}(\zeta)}{F(\zeta)}\right|^{2} d \phi=O\left(\frac{1}{1-R} \log \frac{1}{1-R}\right), \quad R \rightarrow 1
$$

For a proof of (21) see e.g. [2]. Using Lemma 3 and (21) in (19) we have

$$
J_{2}(r) \leqq \frac{A(1-\beta) r^{2(n+1)}}{n^{1 / 2}}\left(\frac{1}{1-r^{-1}} \log \frac{1}{1-r^{-1}}\right)^{1 / 2}, \quad n \geqq 2 .
$$

where $A$ is an absolute constant. Choosing $r=1+1 /(n-1)$ for $n \geqq 2$ we obtain finally from (15), (18) and (22) that

$$
n\left|a_{n}\right|<A_{1}(\log n)^{1 / 2}, \quad n \geqq 2,
$$

and this completes the proof of Theorem 5.

\section{REFERENCES}

1. I. E. Bazilevič, On a case of integrability in quadratures of the Loewner-Kufarev equation, Mat. Sb. 37 (1955), 471-476. (Russian)

2. M. Biernacki, Sur les fonctions en moyenne multivalentes, Bull. Sci. Math 70 (1946), 51-76.

3. J. G. Clunie and C. Pommerenke, On the coefficients of close-to-convex univalent functions, J. London Math. Soc. 41 (1966), 161-165.

4. G. M. Golusin, Geometrische Funktionentheorie, V.E.B. Deutscher Verlag der Wissenschaften, Berlin, 1957.

5. W. K. Hayman, Multivalent functions, Cambridge Univ. Press, Cambridge, 1958.

6. W. Kaplan, Close-to-convex schlicht functions, Michigan Math. J. 1 (1952), 169-185.

7. G. Pólya and G. Szegö, Aufgaben und Lehrsätze aus der Analysis, Springer-Verlag, Berlin, 1925.

8. C. Pommerenke, Uber einige Klassen meromorpher schlichter Funktionen, Math. Z. 78 (1962), 263-284.

9. - On meromorphic starlike functions, Pacific J. Math. 13 (1963), 221-235.

10. - Über die Subordination analytischer Funktionen, J. Reine Angew. Math. 218 (1965), 159-173.

11. D. K. Thomas, On starlike and close-to-convex univalent functions, J. London Math. Soc. 42 (1967), 427-435.

12. J. Zamorski, On Bazilevič schlicht functions, Ann. Polon. Math. 12 (1962), 83-90.

\section{UNIVERSity COLLEGE,}

SWANSEA, UNITED KINGDOM 